\title{
基于深度学习的肾小球病理图像识别与分类
}

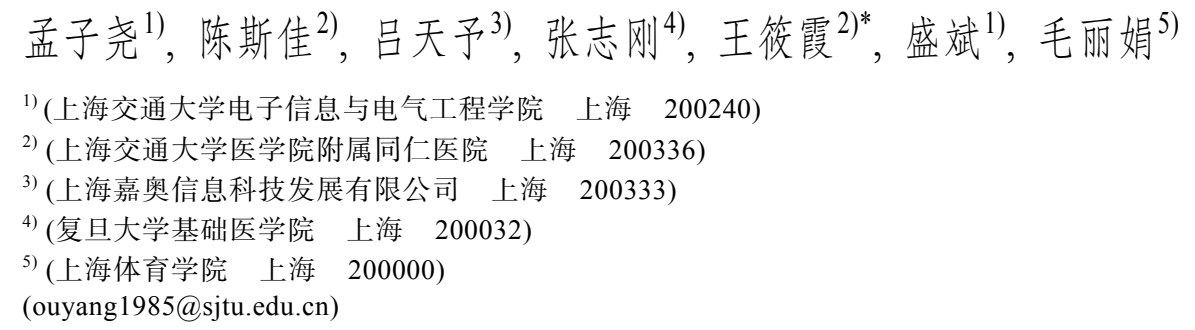

摘 要: 病理切片中肾小球的识别和分类是诊断肾脏病变程度和病变类型的关键, 为解决肾小球的识别和分类问题, 从中检测出肾小球并进行分类，设计了一个基于深度学习的完整的肾小球检测及分类框架. 该框架包括肾小球识别 的 4 个阶段，第 1 阶段的扫描窗生成中，设计一种网络框架 RGNet，用于初步判断肾小球可能出现的位置; 第 2 阶段 的检测和粗分类中, 针对肾小球数据改进了 Faster R-CNN; 第 3 阶段基于 NMS 算法设计了 NMS-Lite 算法, 将检测 到的肾小球进行合并; 在第 4 阶段的细分类中, 使用数据增强等技巧训练 2 个神经网络, 实现肾小球的病变程度分类. 实验结果表明，所提肾小球检测方法在测试集上取得了与同类方法可比的精度，且在一定程度上解决了相似类别的 肾小球难以区分的问题.

关键词: 肾小球病理图像; 病变分类; 神经网络

中图法分类号: TP391.41 DOI: 10.3724/SP.J.1089.2021.18563

\section{Recognition and Classification of Glomerular Pathological Images Based on Deep Learning}

\author{
Meng Ziyao ${ }^{1)}$, Chen Sijia ${ }^{2)}$, Lyu Tianyu ${ }^{3)}$, Zhang Zhigang ${ }^{4)}$, Wang Xiaoxia ${ }^{2}{ }^{*}$, Sheng Bin ${ }^{1)}$, and Mao Lijuan ${ }^{5)}$ \\ 1) (School of Electronic Information and Electrical Engineering, Shanghai Jiao Tong University, Shanghai 200240) \\ 2) (Tongren Hospital, School of Medicine, Shanghai Jiao Tong University, Shanghai 200336) \\ 3) (Keyin Tech, Shanghai 200333) \\ 4) (School of Basic Medical Sciences, Fudan University, Shanghai 200032) \\ 5) (Shanghai University of Sport, Shanghai 200000)
}

\begin{abstract}
The identification and classification of glomeruli in pathological sections is the key to diagnosing the degree and type of renal lesions. In order to solve the problem of glomerular recognition and classification, a complete glomerular detection and classification framework based on deep learning is designed. Glomeruli are detected and classified in the entire slice image. The framework includes four stages of glomerular recognition. In the first stage of scanning window generation, a new network framework, RGNet, is designed to initially deter-
\end{abstract}

\footnotetext{
收稿日期: 2020-08-07; 修回日期: 2020-09-30. 基金项目：国家重点研发计划(2018YFF0300903); 国家自然科学基金(61872241, 61572316); 上海市科教委项目(15490503200, 18410750700, 17411952600). 孟子尧(1998一), 男, 硕士研究生, 主要研究方向为计算机 视觉、机器学习、医学影像识别; 陈斯佳(1995-), 女, 博士研究生, 主要研究方向为线粒体功能障碍在糖尿病肾病发病机制中的作 用; 吕天予(1993-), 男, 学士, 主要研究方向为计算机视觉、医学影像识别; 张志刚(1956一), 男, 博士, 教授, 博士生导师, 主要研 究方向为肾脏病理、间质病理及蛋白质泛素调节与肾小球疾病关系; 王箥霞(1968一), 女, 博士, 主任医师, 博士生导师, 论文通讯作 者, 主要研究方向为表观遗传学在糖尿病肾病中的作用及机制; 盛斌(1981一), 男, 博士, 副教授, 博士生导师, 主要研究方向为计 算机图形学、计算机视觉、机器学习; 毛丽娟(1969-), 女, 博士, 教授, 博士生导师, 主要研究方向为体育教学, 运动训练及不同人 群体质健康.
} 
mine the possible location of glomeruli. In the second stage of detection and coarse classification, Faster R-CNN is improved for glomerular data. In the third stage, the NMS-Lite algorithm is designed based on the NMS algorithm to merge the detected glomeruli. In the fourth stage of fine classification, two neural networks are trained using data augmentation to classify the degree of glomerular lesions. The experimental results has show that the glomerulus detection method proposed in this paper has achieved comparable accuracy on the test set with similar methods, and to a certain extent solves the problem that similar types of glomeruli are difficult to distinguish.

Key words: glomerular pathological image; lesion classification; neural network

近年来，各种计算机视觉的方法，尤其是基于 深度学习的模式识别方法, 已广泛应用于临床医 学图像分析中，包括甲状腺癌笁查、皮肤癌诊断、 眼底图像的异常篮查、基因突变的预测. 通过计算 机辅助医师诊断病理图像，可以节省大量的诊断 时间和人力物力, 给疾病的治疗带来帮助 ${ }^{[1]}$. 随着 计算机设备和数字化影像设备的飞速发展，全视 野数字切片 (whole slide image, WSI)迅速出现在医 学领域中, 数字切片的发展带来了计算机与生物 医学、生物信息学的进一步结合. 其中, 肾小球的 检测和分类是对肾脏切片的组织病理学上的判断 的关键步骤. 根据肾小球的健康状态, 可以判断出 各种肾炎的类别和程度.

本文针对肾小球检测和分类这一特定问题，设 计了一个完整的肾小球识别的方案．整个肾小球识 别的框架如图 1 所示, 分为 4 个阶段, 使用了 4 个 网络. 由于各阶段的输人输出不同，本文对每个阶 段使用分别制作的数据集进行训练和评估. 而由于 数据的分布不均衡, 本文针对数据集的特点对每个 网络使用不同的策略单独进行训练. 其中, 在第 1 阶段的扫描窗生成中，创新性地设计了一种新的网 络框架 RGNet，用于初步判断肾小球可能出现的位 置, 减少之后的计算量; 在第 2 阶段的检测和粗分 类中，针对肾小球数据的特殊性，改进了 Faster $\mathrm{R}-\mathrm{CNN}$; 在第 3 阶段中，基于非极大值抑制(nonmaximum suppression, NMS)算法设计了 NMS-Lite 算法，用于将第 2 阶段检测到的肾小球进行合并; 最后在第 4 阶段的肾小球分类中，使用数据增强等 技巧训练了 2 个神经网络, 在一定程度上解决了相 似类别的肾小球难以区分的问题.

相比现有工作，本文中使用的方法仅用较少 数据就在肾小球检测上取得了可比的结果，在肾 小球定位的平均准确率达到 $89.4 \%$, 平均召回率达 到 $98.2 \%$, 平均 $F$ 值达到 $92.3 \%$. 且本文在现有工

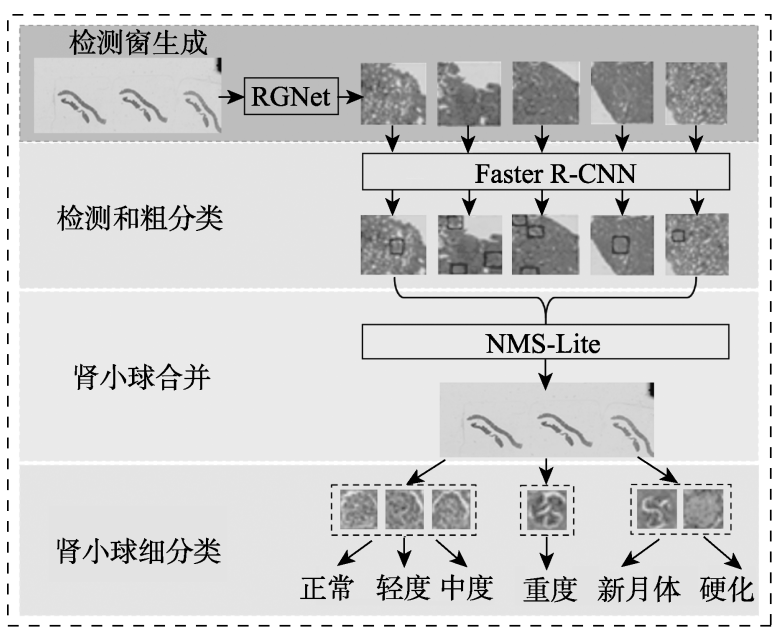

图 1 肾小球识别与分类框架

作肾小球检测的基础上进行了肾小球的细分类这 一已有研究很少涉及的工作，肾小球病变分类的 准确率达到 $80.2 \%$.

\section{1 相关工作}

相关工作主要分为 3 部分: 病理学上关于病理 切片与肾小球病变的相关工作、数字图像处理方面 关于图像分类与目标检测的相关工作以及 2 个领 域相结合的肾小球识别相关工作.

\section{1 病理切片与肾小球病变}

在病理学上，关于肾小球病变及其临床诊断 的方法已经相当成熟. 通过对病理切片的观察，可 以判断其中肾小球的病变类型，进而判断病人肾 炎的类别与程度.

病理切片是病理标本的一种. 制作时将部分 有病变的组织或脏器经过各种化学品和埋藏法的 处理, 使之固定硬化, 在切片机上切成薄片粘附在 玻片上，并染以各种颜色，供在显微镜下检查，以 观察病理变化对其进行诊断, 为临床诊断和治疗 提供帮助. 本文所使用的数据集的染色方式可判 
断为 $\mathrm{HE}$ 染色.

自 1999 年以来, 病理切片向数字化的方向发 展，WSI 就是其产物. WSI 指通过扫描传统的玻璃 切片生成数字切片, 是用于病理学研究的最新的 成像方式 ${ }^{[2]}$. 其中, 本文所使用的数据集的 ndpi 格 式的 WSI 为滨松(HAMAMATSU)公司的成像设备 所产生的.

\section{2 图像分类与目标检测}

计算机视觉中, 目标检测的方法大多基于机 器学习 ${ }^{[3-4]}$. 最初的目标检测方法包括使用方向梯 度直方图 (histogram of oriented gradient, $\mathrm{HOG})^{[5]}$ 、 尺度不变特征变换 (scale-invariant feature transform, SIFT $)^{[6]}$ 等描述符提取图像特征，辅以支持向 量机(support vector machine, SVM) 定位目标. 随着 方法的不断改进, 各种可形变部件模型 (deformable part model, DPM) ${ }^{[7]}$ 进一步利用 HOG 等特征, 将目 标建模为一种弹簧形变模型, 成为了目标检测方 法的主流.

随着以深度学习为代表的特征学习方法的出 现，传统的 hand-crafted 的特征逐渐被替代，取而 代之的是卷积神经网络 (convolutional neural networks, CNN)在图像上提取的更加稳定和可泛化的 特征 ${ }^{[8]}$. 基于深度学习的目标检测算法主要分为两 阶段法和一阶段 2 类.

两阶段的检测算法从图像中提取候选框后，基 于候选区域进一步细化定位和分类. R-CNN ${ }^{[9]}$, Fast $\mathrm{R}-\mathrm{CNN}^{[10]}$ 和 Faster R-CNN ${ }^{[11]}$ 等一系列方法借助 $\mathrm{CNN}$ 的特征实现了准确、高效的目标检测框架，从 用传统的选择性搜索(selective search)生成候选区, 到 2 个阶段使用共享的特征，端到端训练，逐渐发 挥出了深度学习通用性的优势 ${ }^{[12-13]}$. R-FCN ${ }^{[14]}$ 修改 了 Faster R-CNN 的结构, 将感兴趣区域(region of interest，RoI)池化后的层往前移动，同时添加位置 敏感的得分图实现平移可变性，提升了 Faster R-CNN 的速度.

一阶段的检测算法在准确度上略低，但在速 度和资源消耗上较两阶段的算法大大优化. 典型 的一阶段目标检测算法有 YOLO(you only look once) ${ }^{[15]}, \operatorname{SSD}$ (single shot detector) ${ }^{[16]}$ 和特征金字塔 网络(feature pyramid networks, FPN ${ }^{[17]}$. 相较于产 生候选框的二阶段检测算法，一阶段检测算法直 接将目标边框的定位问题转化为回归问题解决. YOLO 算法将图像划分为 $7 \times 7$ 的网格区域，通过 $\mathrm{CNN}$ 提取特征训练后, 直接预测每个网格内的边 框坐标和每个类别置信度. 作为 YOLO 改进版的
YOLO v2, 借鉴了 Faster R-CNN, 引入针(anchor) 机制, 结合图像细粒度特征, 增强了对小尺寸目标 的检测. SSD 算法结合 YOLO 的回归思想和 Faster R-CNN 的 anchor box 方法, 可以直接预测边框 (bounding box)的坐标和类别, 通过在不同卷积层 的特征图上预测物体区域，可以针对不同尺寸的 物体进行检测，但其对小目标检测困难. FPN 算法 同样是利用不同卷积层的特征图进行不同尺寸的 目标预测，其特别之处在于预测是在不同特征层 独立进行的，将语义信息较强的深层特征图和分 辨率较高的浅层特征图相结合.

在医疗图像识别中，对于目标检测算法的实 时性要求不高, 通常使用两阶段的目标检测算法, 以获取更高的检测准确率和定位精度.

\section{3 肾小球识别}

现有的医疗图像识别的方法很多, 但主要集 中在血管分割等方面, 而在肾小球识别上可参考 的工作较少. 其中, S-HOG(segmental HOG) ${ }^{[18]}$ 针对 肾小球的外观特征，修改自传统的 R-HOG. 用 SVM 分类时, 使用 S-HOG 的召回率略微降低, 但 准确率较 R-HOG 提高了 $10.0 \%$. Pedraza 等 ${ }^{[19]}$ 使用 预训练的 AlexNet 模型从切片中检测肾小球, 基于 一个包含 10 幅 WSI 和 275 个肾小球的数据集, 其 检测准确率为 $88.1 \%$, 召回率为 $100.0 \%$. Zhao 等 ${ }^{[20]}$ 在整个肾组织的显微照片上提出了一种全自动肾 小球提取框架, 重点是提取鲍曼氏囊(肾小球的支 撑结构), 并引人统计方法进一步提高性能, 通过 适当的调整策略，准确率可以达到 $90.0 \%$. Simon 等 ${ }^{[21]}$ 采用局部二值模式图像特征向量训练 SVM 模 型，检测 WSI 中的肾小球. 该方法对多种物种(大 鼠、小鼠和人类)、多种载玻片染色方法都有着较 强的适应性, 同时具备较高的准确率 $(>90.0 \%)$ 与 合理的召回率 $(>70.0 \%)$.

\section{2 数据集处理}

本文的原始数据集共有含 11 幅切片的文件及 相应的标注文件, 大小从 $286 \mathrm{MB}$ 到 $2.4 \mathrm{~GB}$ 不等. 在切片文件中, $x$ 和 $y$ 轴上的一像素点对应实际大 小的约 $0.227 \mu \mathrm{m}$. 载玻片上 $1 \mathrm{~cm} \times 1 \mathrm{~cm}$ 的区域, 对 应的切片图像大小为 $44128 \times 44128$ 像素. 该批数 据一共有 7 类肾小球标注，分别是正常、新月体、 轻度、中度、重度、硬化与未知.

\section{1 数据集制作}

由于算法分为检测窗生成、检测和粗分类、合 
并、细分类等 4 个阶段，单一数据集无法满足不同 阶段训练的需要. 因此, 本文共制作了 4 个数据集. 分别是单一肾小球数据集、肾小球九宫格数据集、 目标检测数据集和 WSI 数据集.

单一肾小球数据集是为了完成肾小球分类任 务. 此数据集的格式处理较为简单, 仅需从原始切 片中根据标注文件中的坐标信息和类别信息，提 取出每一个肾小球并保存.

肾小球九宫格数据集与单一肾小球数据集较 为类似. 单一肾小球数据集中, 单幅图像仅包含肾 小球区域，不包含肾小球的周围区域.

目标检测数据集须设置一个滑窗，遍历整幅 图像. 每当滑窗移动到一个新的位置, 都根据读取 标注文件中的信息, 判断当前滑窗是否含有标注 过的肾小球. 若无，则不保存该区域的图像，移动 到下一位置; 否则, 为了方便训练, 应该尽可能地 让滑窗包含更多肾小球的区域, 且应避免出现单 幅图像中仅边缘位置出现肾小球的情况.

11 幅原始切片制作出来的数据集图像数量分 别为 $28,33,73,85,106,124,181,275,404,610$, 828. 数据集需要划分为训练集与测试集, 划分过 程不能简单地根据切片来划分，否则会出现各类 肾小球数量严重不平衡的问题. 在划分数据集前, 手动将单幅切片划分为左右 2 个区域，左侧区域 中裁取出的图像作为训练集, 右侧区域裁取出的 图像作为测试集，共 2747 幅图像及其对应的标注 文件.

WSI 数据集是为检测窗生成阶段而准备. 本 文将原始的 11 幅切片进行 4 倍的下采样并保存.

\section{2 数据集扩充}

为了进一步扩充数据集, 如表 1 所示采取 3 种 数据增强的手段旋转、色彩抖动和 mixup. 位置变 换结合水平翻转、垂直翻转和逆时针旋转 $90^{\circ}$, 作 为对原始图像进行位置变换的手段, 这样最多可 以将数据集增加到原来的 8 倍大小. 由于肾小球的 分类主要以形态为标准, 色彩信息对其影响不大, 且不同切片间的色调可能存在较大差异. 色彩变 换选择对图像的亮度、对比度、色相和饱和度等进 行随机抖动, 增加模型的泛化能力. mixup 将数据 集中随机抽取的 2 个样本的图像和标签分别进行 混合, 从而构建出新的虚拟样本.

(1) 旋转. 肾小球的类别与它的位置、角度无 关, 因此应该可以任意旋转而不改变类别. 根据数 据的类型不同，旋转又可分为轴对齐的旋转和任 意的旋转. 在紧密包裹着一个肾小球的图像中, 只
表 1 图像增强示例

\begin{tabular}{llll}
\hline 增强方式 & 原始样本 & 虚拟样本 \\
\hline 旋转 & & & \\
& & & \\
mixup & & &
\end{tabular}

能采用轴对齐的旋转, 通过随机的水平翻转、随机 的垂直翻转和随机旋转 $90^{\circ}$, 一幅图像可以扩展为 8 份. 而在周围有边界的肾小球图像中, 可以对图 像旋转任意的角度, 然后从图像中央将肾小球裁 剪出来.

(2) 色彩抖动. 经观察, 11 幅切片的染色方法 都相似或相同, 但是染色的深浅程度、成像的亮度 都有很大的差别. 进一步, 每幅切片中出现的肾小 球种类有偏差, 如有的切片中大多数为正常肾小 球，而有的切片中大多数为重度系膜增生的肾小 球; 这样导致有的肾小球仅通过颜色就能被分类, 尽管肾小球的类别与颜色无关. 为了防止网络学 习到无关的颜色信息, 本文使用了随机的色彩抖 动作为数据增强. 训练集数据输人网络前, 会随机 地调整饱和度、对比度和色相, 其中饱和度抖动的 范围为 $50 \%$, 对比度为 $20 \%$, 色相为 $5 \%$.

（3） mixup. mixup 也是一种数据增强方法, 它 将数据集中随机抽取的 2 个样本的图像和标签分 别进行混合，从而构建出新的虚拟样本. 图像混合 的比例遵循 $\beta$ 分布, 即 $\lambda \sim \beta(\alpha, \alpha)$. $\alpha$ 越大, mixup 的强度越大, 当 $\alpha=0$ 时, 相当于不使用 mixup. 尽管 mixup 效果很好, 但混合得到的虚拟 样本通常是违背常理的, 然而在对肾小球系膜增 生程度分级的任务上, mixup 得到的样本在视觉上 更加合理地反映了不同分级之间的连续变化.

\section{3 肾小球识别与分类框架}

\section{1 检测窗生成}

检测窗生成使用了一个扫描窗，对整幅 WSI 进行了遍历. 通过观察数据集发现 WSI 上经常有 大量的留白, 如果仍然使用扫描窗遍历, 必然会造 成大量的时间浪费.

为提高检测窗生成的性能，本文借鉴 YOLO 的检测方法，提出了名为区域网格网络(region grid 
networks，RGNet)的检测窗生成方法，其工作流程 如图 2 所示. 该网络的核心思想为将检测窗可能的 位置限定为网格，不同于扫描窗遍历方法将网格 上的位置都使用一次, RGNet 使用一个全卷积网络 (fully convolutional networks, FCN) 预测每个网格 内出现肾小球的概率，并将这些网格组合成检测 窗，供 Faster R-CNN 检测.

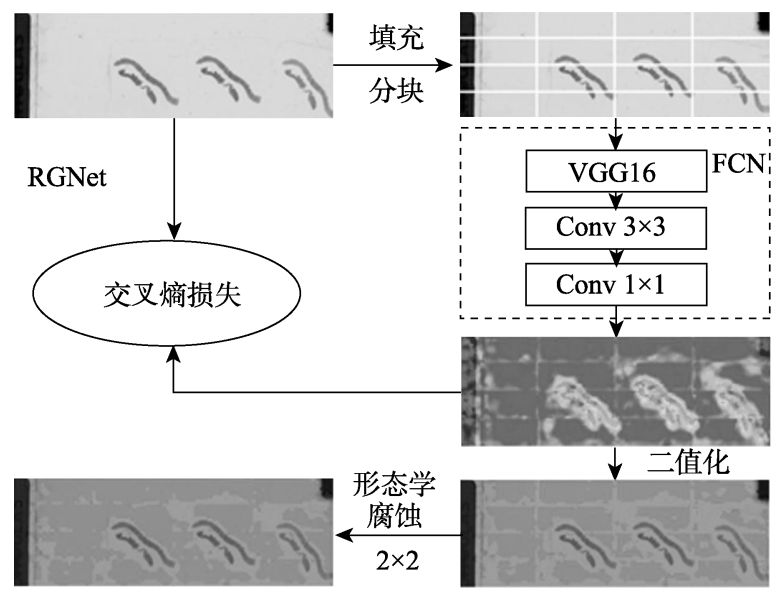

图 2 RGNet工作流程

对于使用 $3000 \times 3000$ 的检测窗，网格大小被设 定为 $1500 \times 1500$. 首先, 对于输人的 WSI 图像, 为 了保证其能分成所需的网格，RGNet 对其边缘进行 填充, 使图像的长和宽都为 1500 的倍数. 其次, 由 于数据量较少，整幅图像被分为 $4 \times 4$ 块进行训练和 测试, 这样就得到了 16 倍的数据. 训练时, 每一块 图像分别被输人网络，而在测试时，一幅 WSI 的 16 块图像作为一批同时被输人网络. 网络由 VGG16 的卷积层和另外 2 个卷积层组成, 共 18 层, 最后输 出的通道数为 2 . 之后的流程分为训练和测试.

训练时，对于每个网格，它的标签为是否有肾 小球的中心落在其中. 每个网格上的预测值与其 标签进行计算，产生一个交叉熵损失，用于更新网 络权重. 由于数据量小, 本文只在训练集上将 RGNet 训练了 2 代，以防过拟合.

测试时, 网络输出的 Softmax 值被作为每个网 格的分数. 由于训练时正负样本不均, 网络预测网 格包含肾小球的概率往往低于不包含肾小球的概 率, 因此本文不直接使用分数是否大于 0.5 作为判 断, 而是选取一定比例的分数最高的网格. 随后, 若相邻的 $2 \times 2$ 个网格都被预测为包含肾小球，则将 这 4 个网格组成一个 $3000 \times 3000$ 的检测窗并输出.

值得注意的是, 如果将每个网格看做一个像 素, 那么选取网格的过程可以看做网格图像的二
值化，从网格中选取检测窗的过程可以看做在网 格上的形态学的腐蚀操作, 使用的结构元的大小 为 $2 \times 2$, 因此用图像处理的算法可以很方便地实现 整个检测窗生成的框架.

\section{2 肾小球检测}

在整幅切片中，肾小球的位置并不确定，为此 需要先将肾小球定位后才能进行分类. 本文检测 方法使用的是 Faster R-CNN.

本文实现了 Faster R-CNN 的网络结构及其训 练和测试流程. 实现的流程和思路如图 3 所示.

本文首先尝试仅使用 R-CNN 在肾小球数据上 对一个肾小球定位，发现定位的效果很好，而最终 定位准确率主要受限于分类的准确率. 因此, 为了 将主要任务放在检测和定位上, Faster R-CNN 只将 肾小球粗分类为 3 大类：正常、轻度或中度系膜增 生肾小球，重度系膜增生肾小球以及新月体、硬化 肾小球; 而将细分类的任务留给后文提讨论的 2 个 分类网络完成. 检测准确率的评价指标有准确率、 召回率、 $F$ 值和平均精度均值(mean average precision, $\mathrm{mAP}$ ).

数据集中存在相当数量的未知类别的肾小球, 如果在训练过程中忽略它们, 则一方面浪费了这 些标注, 另一方面可能在负样本中混人肾小球, 导 致网络学习到错误的背景信息. 针对这种情况, 在 使用这些未知肾小球的标注的同时, 本文修改了 Faster R-CNN 的损失函数. 简单来说，未知类别的 肾小球被标注为 -1 , 训练时这个类别从 R-CNN 的 分类损失中被剔除. 同时，在评价检测准确率时， 未知类别的真值 (ground-truth)可以匹配网络预测的 任何类别的肾小球. 后面的结果中将会展示使用未 知类别的肾小球进行训练可以提高准确率约 $2.0 \%$.

肾小球检测数据集随机划分为训练集(共 2247 幅图像)和验证集(共 500 幅图像). 训练和测试时都 不使用只出现 $30 \%$ 面积的肾小球. 在训练时, 类似 肾小球分类, 数据增强中采用了翻转和色彩抖动 (抖动幅度仅为之前的一半) 2 种手段. 训练首先使 用 0.001 的学习率训练 12 代 (约 20000 次迭代), 然 后用 0.0001 的学习率训练 12 代, 最后用 0.00001 的学习率训练 24 代至收玫. 最终训练集和验证集 上的准确率差距不超过 $5.0 \%$.

\section{3 肾小球合并}

在各个检测窗中检测到的肾小球之间会存在 重叠, 因此在检测完成后还要进行一次合并. 合并 过程使用非极大值抑制(non-maximum suppression, NMS)算法即可完成，但针对本文肾小球检测任务 


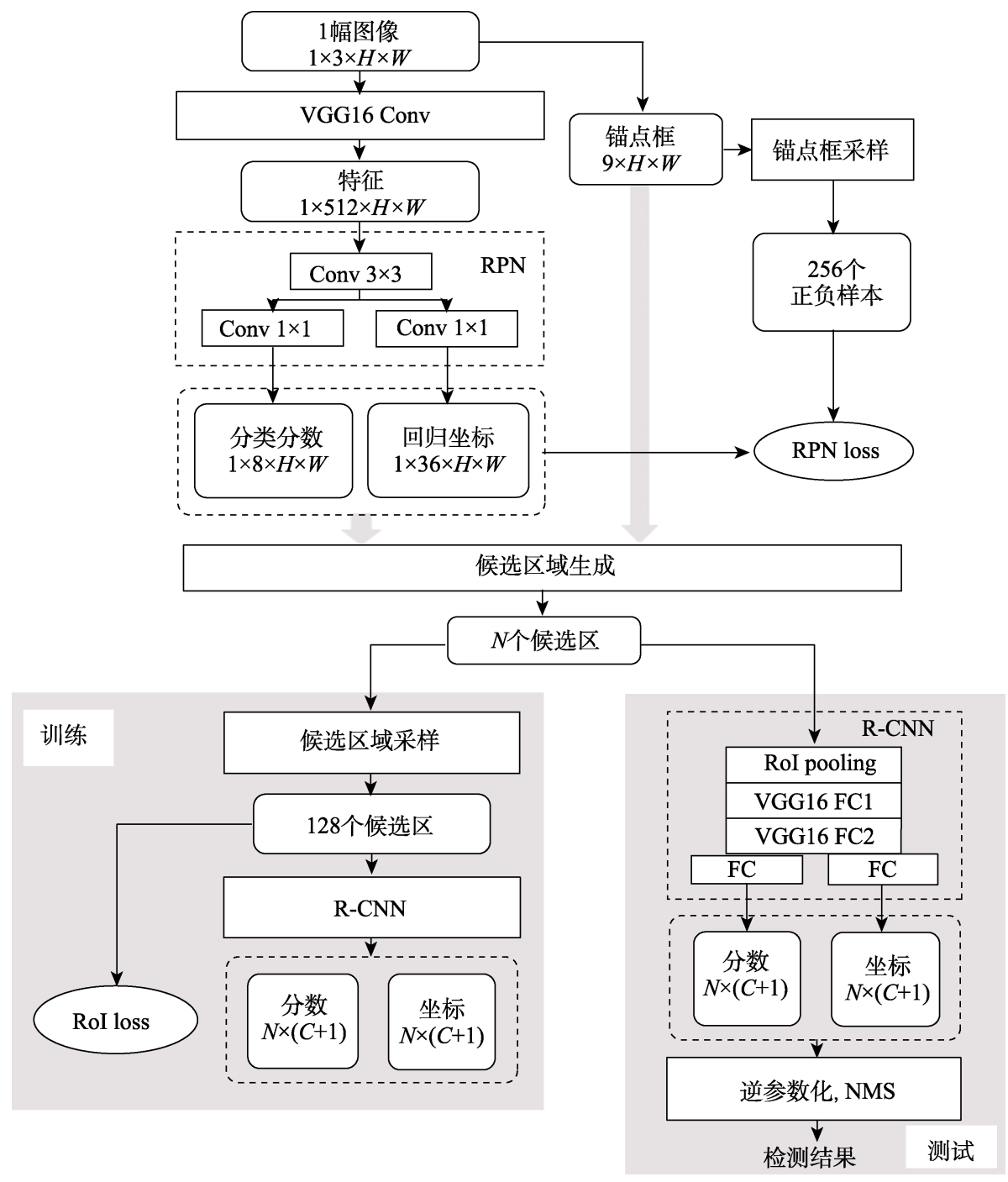

图 3 本文采用的 Faster R-CNN 的结构

的实际情况, 将其改进为 NMS-Lite 算法.

(1) 类间抑制. 传统的 NMS 算法只对相同类 别的窗口(bounding box)进行抑制, 而实际情况下 同一个位置不会重叠地出现 2 个不同类别的肾小 球. 尽管重复的窗口不会对召回率和 $\mathrm{mAP}$ 等评价 指标产生影响, 但是为了使合并后的结果更加清 晰, NMS-Lite 中对不同类别间的肾小球也进行抑 制，防止出现同一位置检测出 2 种肾小球的情况.

（2）分块抑制. 传统的 NMS 算法在每个循环 会检查所有窗口与一个窗口间的重叠率，然而在 对检测窗合并时，只有相邻检测窗间可能有重叠 的窗口. 因此，在 NMS-Lite 中，窗口分批进行处 理, 每次只在相邻的 $2 \times 2$ 个检测窗中抑制窗口. 这 样虽然不会减少 NMS 的时间复杂度，但理论上可 以将常数缩小.

(3) 边缘抑制. 如图 4 所示, 由于一个肾小球 可能在多个检测窗(最多 9 个)中被检测到, 其中
有的检测窗中虽然能检测到这个肾小球, 但检测 到的不是完整的肾小球，从而窗口小于完整的肾 小球. 进而, 完整的窗口与残缺的窗口之间的重 叠率会低于阈值，在使用传统 NMS 算法合并检测 窗时，残缺的窗口不会被剔除. 为解决这个问题, 在 NMS-Lite 算法中会将一个检测窗中的窗口裁 剪到相邻检测窗中，尝试对相邻检测窗中的窗口 进行抑制, 以避免出现如图 4 右侧例子中重叠窗 口的情况.

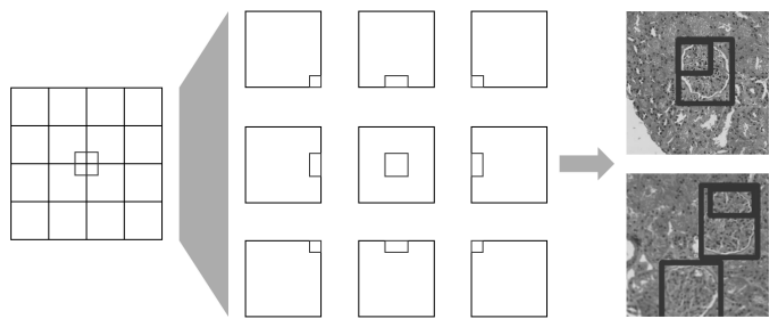

图 4 合并检测窗时可能出现的问题 


\section{4 肾小球分类}

根据肾小球的类别分布和视觉特征，首先将 肾小球粗分类为 3 类. 正常肾小球和轻度、中度系 膜增生的肾小球由于看起来非常相似，相互之间 难以被人或分类器区分，因此被划为一类. 新月体 和硬化同属于特殊类别, 且硬化数据量极小, 需要 单独处理，因此将这 2 种肾小球合并为一类. 重度 系膜增生的肾小球由于特征明显，容易区分，因此 单独作为一类. 通过先将肾小球粗分为 3 个大类, 再向下细分为 6 个小类，由此构建了一种类似决策 树的模型. 粗分类使用 CNN 可以轻松达到 $97.0 \%$ 以上的准确率，相比之下细分类要困难得多.

对正常、轻度、中度分类，难点在于 3 类的可 分性很差. 举例说明, 使用 CNN 对正常和轻度 2 类肾小球进行二分类时，网络非但在验证集上的 准确率难以达到 $60.0 \%$, 而且在训练集上也几乎无 法拟合数据, 说明了分类的困难. 产生这种情况的 原因是肾小球增生程度的判断依据是肾小球内的 系膜组织和毛细血管的特征，然而肾小球内的毛 细血管和细胞核并没有标注，只能依靠分类模型 自动学习, 而模型的表达能力和学习能力不够强, 且不同类别的数据之间差异过小, 使分类器拟合 数据非常困难.

为了给这 3 类分类, 本文发现与从头训练神经 网络相比, 使用预训练的神经网络和较大的初始 学习率可以很快地拟合数据. 在使用预训练的较 深的 ResNet-101 模型时, 训练集准确率可以在 20 代内达到 $99.0 \%$ 以上，而从头开始训练即使 200 代 以后也只能达到 $80.0 \%$. 通过使用预训练模型, 可 以解决网络不收玫的问题.

然而, 神经网络具有很强的记忆数据的能力, 甚至可以拟合噪声图像和随机打乱的标签. 当这 种记忆能力超过了网络自身的泛化能力, 就会产 生一种过拟合. 因此, 在保证网络能拟合训练集数 据的前提下，还要通过减小过拟合来保证验证集 和测试集上的准确率. 本文采用第 2.2 节提出的数 据增强方法来应对这一问题.

\section{4 实验结果与分析}

肾小球分类在验证集上的准确率分别为粗分 类为 $97.6 \%$, 正常、轻度和中度细分类为 $66.7 \%$ ，硬 化和新月体分类为 $88.3 \%$.

Faster R-CNN 的训练结果如表 2 所示. 可以看 出, 本文提出的通过修改 Faster R-CNN 的损失利
用未知类别的标注对结果产生了很大帮助, 数据 增强的策略也起到了一定帮助。

表 2 Faster R-CNN 在测试数据集上的 mAP \%

\begin{tabular}{lcc}
\hline \multirow{2}{*}{ 模型 } & \multicolumn{2}{c}{ 验证集 } \\
\cline { 2 - 3 } & 不包含未知类 & 包含未知类 \\
\hline Baseline & 83.5 & 82.1 \\
+使用未知类训练 & 85.8 & 84.3 \\
+颜色抖动 & 86.5 & 85.4 \\
\hline
\end{tabular}

本文在测试集上进行了肾小球的识别测试, 并与同类方法进行比较, 结果如表 3 所示. 由于先 前的工作只对肾小球进行了检测而没有分类，为 了评价的公平性，本文也只评价检测出肾小球的 准确率和召回率, 而不考虑肾小球的类别. 最终仅 保留置信度大于 $95.0 \%$ 的肾小球作为检测结果. 在 使用数据量远低于其他方法的情况下, 本文方法 的检测准确率能与其他方法持平.

整个框架的检测结果的示例在图 5 中展示. 不 难看出, 由于本文使用了较小的检测窗, 检测的召 回率比较高. 然而, 检测结果出现了较多的假阳 性, 且大多出现在切片边缘的组织上; 而由于这些

表 3 不同方法在测试集上的性能比较

\begin{tabular}{|c|c|c|c|c|}
\hline \multirow{2}{*}{ 方法 } & \multirow{2}{*}{ WSI 数量 } & \multicolumn{3}{|c|}{ 评价指标/\% } \\
\hline & & 召回率 & 准确率 & $F$ 值 \\
\hline $\mathrm{R}-\mathrm{HOG}+\mathrm{SVM}^{[18]}$ & \multirow{2}{*}{80} & & & $40.5 \sim 55.1$ \\
\hline $\mathrm{CNN}$ & & & & $52.2 \sim 71.6$ \\
\hline $\mathrm{CNN}^{[19]}$ & 108 & 100.0 & 88.1 & 93.7 \\
\hline $\operatorname{mrcLBP}+\mathrm{SVM}^{[21]}$ & 25 & 76.1 & 91.7 & 83.2 \\
\hline Faster R-CNN ${ }^{[22]}$ & 200 & 91.9 & 93.1 & 92.5 \\
\hline $\begin{array}{l}\text { RGNet+Faster } \\
\text { R-CNN(本文) }\end{array}$ & 11 & 98.2 & 87.1 & 92.3 \\
\hline
\end{tabular}

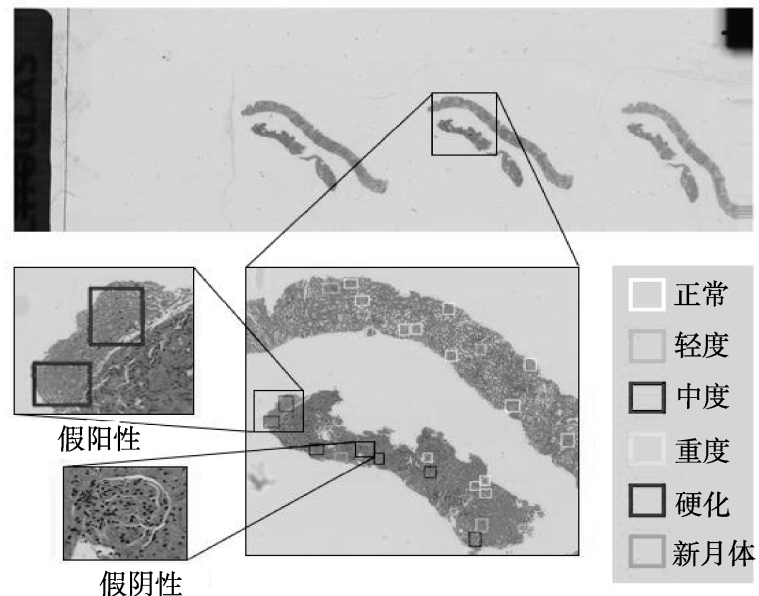

图 5 本文检测结果及部分错误示例 
组织没有明确的圆形轮廓, 上面的图像经常会被 识别为硬化肾小球. 本文认为这是由于训练 Faster R-CNN 的样本只包括了包含肾小球的区域，因此 Faster R-CNN 未使用边缘区域的负样本进行训练.

\section{5 结 语}

本文提出了一个肾小球识别的方案，其中包 括检测窗生成、肾小球检测和粗分类、肾小球合并 和肾小球细分类 4 个阶段. 考虑数据集中数据数量 少且分布不均衡的特点, 这 4 个阶段独立地进行设 计、训练和测试, 最终再进行合并. 对于每个阶段 的训练和测试，本文都分别为之设计了相应的数 据集，使各个阶段都能更加高效地发挥出其应有 的性能.

在检测窗生成阶段，本文提出了名为 $\mathrm{RGNet}$ 的检测窗生成框架，快速地篮选可能出现肾小球 的区域, 并将这些区域提供给检测网络. 在检测肾 小球阶段, 本文修改了 Faster R-CNN 的损失函数, 使网络可以充分利用数据集中未标注类别的肾小 球. 在合并 Faster R-CNN 检测结果阶段, 本文优 化了传统的 NMS 算法，优化后的 NMS-Lite算法对 于合并肾小球检测结果速度更快, 准确度更高. 在 肾小球分类阶段，本文使用了先粗分类、后细分类 的类似决策树的方法, 先利用 Faster R-CNN 将肾 小球粗分类为 3 大类，而后可以将重点放在特殊的 类别和区分度小以致难以分类的类别上.

然而，4 个阶段分别进行的问题在于，前面阶 段的错误会累积到后面阶段. 例如，检测窗生成时 如遗漏了检测窗，则 Faster R-CNN 就不会检测到 那个位置的肾小球; 而如果 Faster R-CNN 粗分类 时出现分类错误，则后面细分类的结果也一定是 错的. 此外, 由于检测在每个检测窗内独立进行, 分类对每个肾小球独立进行, 因此能使用的上下 文信息也是有限的. 在数据量充足的情况下，如果 能实现整个肾小球识别框架的端到端学习, 辅以 不同分辨率的图像的特征融合, 性能可能会进一 步提升.

未来工作中，本文需要对检测数据集的选取 进行改进. 直接从整幅 WSI 中随机采样是不可取 的，一是因为其中有大量留白，会导致网络大部分 时间在空白区域训练，抓不住重点区域; 二是因为 数据集中存在未标注的肾小球, 直接采样可能会 带来假的负样本. 因此，改进的方案可以是利用 RGNet 输出的结果, 从中选取检测窗, 将数据集集
中在图像上容易混淆的地方上，而不是空白的区 域上.

\section{参考文献(References):}

[1] Zhao Mengmeng, Wang Yang, Deng Jiajun, et al. Research progress of artificial intelligence convolutional neural network in whole slide image analysis[J]. Chinese Journal of Clinical Thoracic and Cardiovascular Surgery, 2019, 26(11): 1063-1068 (in Chinese)

(赵蒙蒙, 汪洋, 邓家骏, 等. 人工智能卷积神经网络在全视 野数字切片图像分析中的应用进展 $[\mathrm{J}]$. 中国胸心血管外科 临床杂志, 2019, 26(11): 1063-1068)

[2] Ghaznavi F, Evans A, Madabhushi A, et al. Digital imaging in pathology: whole-slide imaging and beyond[J]. Annual Review of Pathology: Mechanisms of Disease, 2013, 8: 331-359

[3] Lu P, Sheng B, Luo S M, et al. Image-based non-photorealistic rendering for realtime virtual sculpting[J]. Multimedia Tools and Applications, 2015, 74(21): 9697-9714

[4] Kamel A, Sheng B, Yang P, et al. Deep convolutional neural networks for human action recognition using depth maps and postures[J]. IEEE Transactions on Systems, Man, and Cybernetics: Systems, 2019, 49(9): 1806-1819

[5] Dalal N, Triggs B. Histograms of oriented gradients for human detection[C] //Proceedings of the IEEE Computer Society Conference on Computer Vision and Pattern Recognition. Los Alamitos: IEEE Computer Society Press, 2005, 1: 886-893

[6] Lowe D G. Distinctive image features from scale-invariant keypoints[J]. International Journal of Computer Vision, 2004, 60(2): $91-110$

[7] Felzenszwalb P F, Girshick R B, McAllester D, et al. Object detection with discriminatively trained part-based models[J]. IEEE Transactions on Pattern Analysis and Machine Intelligence, 2009, 32(9): 1627-1645

[8] Zhang P, Zheng L H, Jiang Y, et al. Tracking soccer players using spatio-temporal context learning under multiple views[J]. Multimedia Tools and Applications, 2018, 77(15): 1893518955

[9] Girshick R, Donahue J, Darrell T, et al. Rich feature hierarchies for accurate object detection and semantic segmentation[C] //Proceedings of the IEEE Conference on Computer Vision and Pattern Recognition. Los Alamitos: IEEE Computer Society Press, 2014: 580-587

[10] Girshick R. Fast R-CNN[C] //Proceedings of the IEEE International Conference on Computer Vision. Los Alamitos: IEEE Computer Society Press, 2015: 1440-1448

[11] Ren S Q, He K M, Girshick R, et al. Faster R-CNN: towards real-time object detection with region proposal networks[J]. IEEE Transactions on Pattern Analysis and Machine Intelligence, 2017, 39(6): 1137-1149

[12] Karambakhsh A, Kamel A, Sheng B, et al. Deep gesture interaction for augmented anatomy learning $[\mathrm{J}]$. International Journal of Information Management, 2019, 45: 328-336

[13] Sheng B, Li P, Zhang Y H, et al. GreenSea: visual soccer analysis using broad learning system[J]. IEEE Transactions on 
Cybernetics. 2021, 51(3): 1463-1477

[14] Dai J F, Li Y, He K M, et al. R-FCN: object detection via region-based fully convolutional networks[OL]. [2020-08-07]. https://arxiv.org/abs/1605.06409

[15] Redmon J, Divvala S, Girshick R, et al. You only look once: unified, real-time object detection[C] //Proceedings of the IEEE Conference on Computer Vision and Pattern Recognition. Los Alamitos: IEEE Computer Society Press, 2016: 779-788

[16] Liu W, Anguelov D, Erhan D, et al. SSD: single shot multibox detector[C] //Proceedings of European Conference on Computer Vision. Heidelberg: Springer, 2016: 21-37

[17] Lin T Y, Dollár P, Girshick R, et al. Feature pyramid networks for object detection[C] //Proceedings of the IEEE Conference on Computer Vision and Pattern Recognition. Los Alamitos: IEEE Computer Society Press, 2017: 2117-2125

[18] Kato T, Relator R, Ngouv H, et al. Segmental HOG: new descriptor for glomerulus detection in kidney microscopy im-
age[J]. BMC Bioinformatics, 2015, 16(1): Article No.316

[19] Pedraza A, Gallego J, Lopez S, et al. Glomerulus classification with convolutional neural networks[C]//Proceedings of Annual Conference on Medical Image Understanding and Analysis. Heidelberg: Springer, 2017: 839-849

[20] Zhao Y, Black E F, Marini L, et al. Automatic glomerulus extraction in whole slide images towards computer aided diagnosis[C] //Proceedings of the 12th IEEE International Conference on e-Science. Los Alamitos: IEEE Computer Society Press, 2016: $165-174$

[21] Simon O, Yacoub R, Jain S, et al. Multi-radial LBP features as a tool for rapid glomerular detection and assessment in whole slide histopathology images[J]. Scientific Reports, 2018, 8(1): Article No.2032

[22] Kawazoe Y, Shimamoto K, Yamaguchi R, et al. Faster R-CNN-based glomerular detection in multistained human whole slide images[J]. Journal of Imaging, 2018, 4(7): 91 